\title{
Tendencia de la enfermedad diarreica aguda (EDA) en niños menores de cinco años de edad en la Región Amazonas - Perú, 2000 - 2017
}

\author{
Trend of acute diarrheal disease (ADD) in children under five years of age, in the \\ Amazon Region - Perú, 2000-2017
}

\author{
Mariel del Rocío Chotón Calvo ${ }^{1}$
}

\section{RESUMEN}

Se realizó un estudio descriptivo longitudinal con el objetivo de determinar la tendencia de la enfermedad diarreica aguda (EDA) en niños menores de cinco años de edad, en la Región Amazonas y en la Provincia de Chachapoyas, durante los años 2000 a 2017, considerando su tipo acuosa y disentérica; para los cual se contó con información de la Dirección Regional de Salud de Amazonas (DIRESA), considerando los registros de los casos presentados en ese periodo de tiempo, obteniéndose tablas, gráficos, y la tasa de prevalencia anual por cada mil infantes. Los resultados muestran que en la Región Amazonas la tasa de prevalencia de la EDA en niños menores de cinco años de edad tuvo tendencia decreciente desde el año 2004 hasta el año 2017, la EDA acuosa tuvo tendencia decreciente a partir del año 2010 con excepción del año 2014 que se incrementó, la EDA disentérica tuvo una tendencia decreciente para el año 2017 con 58,5 episodios por cada 1000 infantes. Para la provincia de Chachapoyas durante los años 2000 al 2017, la EDA tuvo tendencia decreciente a partir del año 2015 hasta el 2017, la acuosa fue decreciente en el año 2017, y la disentérica también fue decreciente hasta el 2015, luego del cual aumentó.

Palabras claves: enfermedades, infancia, tendencia.

\begin{abstract}
A longitudinal descriptive study was carried out with the objective of determining the trend of acute diarrheal disease (ADD) in children under five years of age, in the Amazon Region and in the Chachapoyas Province, during the years 2000 to 2017, considering its aqueous and dysenteric type; for which information was available from the Regional Health Directorate of Amazonas (DIRESA), considering the records of the cases presented in that period of time, obtaining tables, graphs, and the annual prevalence rate per thousand infants. The results show that in the Amazon Region the prevalence rate of EDA in children under five years of age had a decreasing trend from 2004 to 2017, the aqueous EDA had a decreasing trend as of 2010 with the exception of the year 2014 that increased, the dysenteric EDA had a decreasing tendency for the year 2017 with 58.5 episodes per 1000 infants. For the province of Chachapoyas during the years 2000 to 2017, the EDA had a decreasing trend from 2015 to 2017, the watery one was decreasing in the year 2017, and the dysenteric one was also decreasing until 2015, after which it increased.
\end{abstract}

Keywords: diseases, childhood, tendency. 


\section{INTRODUCCIÓN}

La salud de las personas en general, y la de las niñas y niños en particular es un derecho humano inalienable puesto en evidencia en la Convención sobre los Derechos del Niño (Resolución 44/25, Naciones Unidas, Ginebra, noviembre 1989) y ratificado como tal en los instrumentos legales de cada país. Esto supone ser obligatorio y su vigilancia y exigencia constantes. El estudio de los problemas de salud es necesario para comprender la compleja y diversa realidad social, lo cual permitirá conocer acerca de las enfermedades que afectan a la población y permitirá tomar decisiones en el campo de la salud, permitiendo nuevas y mejores oportunidades a las personas, familias y comunidades en temas de salud y prevención. (OPS, 2009)

Las enfermedades diarreicas son una causa principal de mortalidad y morbilidad en la niñez, a nivel mundial se producen 1700 millones de casos de enfermedades diarreicas cada año, y constituye una de las principales causas de malnutrición de niños menores de cinco años, y por lo general son consecuencia de la exposición a alimentos o agua contaminados, ya que se conoce que en todo el mundo alrededor de 780 millones de personas carecen de acceso al agua potable, y 2500 millones a sistemas de saneamiento apropiados, por lo que en países en desarrollo, la diarrea es causada por infecciones. (OMS, 2013)

Pese a que las enfermedades diarreicas agudas son enfermedades prevenibles y tratables, estas matan a 525000 niños menores de cinco años cada año, pudiéndose prevenir mediante el acceso al agua potable y a servicios adecuados de saneamiento e higiene. (OMS, 2013)

Los episodios de EDA (enfermedad diarreica aguda) reportados en el año 2016 fue de 15,4\% a nivel nacional y de 24,8\% para la Región Amazonas, observándose un decremento con respecto al año 2015 cuya tasa fue de $26,3 \%$ pero aún sigue siendo alto si se compara con el promedio nacional. (MINSA, 2016)

Los resultados del presente estudio están orientados a proporcionar información de la tendencia de la enfermedad diarreica aguda (EDA) en niños menores de un año de edad a nivel de la región Amazonas y conocer su comportamiento numérico lo cual permitirá analizar su tendencia, constituyendo una herramienta indispensable para orientar adecuadamente la realización de que una adecuada planificación de la gestión de los servicios de salud, para la priorización de los principales problemas de salud que afectan a la población de niños menores de una año de edad, así como para la adecuada toma de decisiones.

\section{MATERIAL Y MÉTODO}

Tipo y diseño de investigación:Descriptiva, longitudinal, ambispectivo, cuyo diseño es:
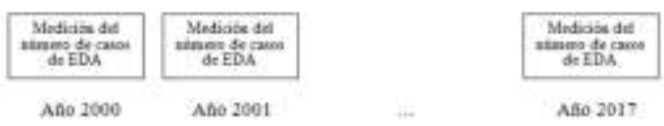

Población-Muestra: Estuvo constituida por los niños menores de cinco años de edad:

\begin{tabular}{|l|c|c|}
\hline \multirow{2}{*}{ Años } & \multicolumn{2}{|c|}{$\mathbf{N}^{\circ}$ niños } \\
\cline { 2 - 3 } & $<\mathbf{1}$ año & $\begin{array}{c}<\text { años de } \\
\text { edad }\end{array}$ \\
\hline 2005 & 12711 & 62732 \\
\hline 2006 & 12725 & 62781 \\
\hline 2007 & 10676 & 53756 \\
\hline 2008 & 10449 & 53002 \\
\hline 2009 & 10206 & 52103 \\
\hline 2010 & 9623 & 47969 \\
\hline 2011 & 9643 & 47500 \\
\hline 2012 & 9389 & 46837 \\
\hline 2013 & 9085 & 46043 \\
\hline 2014 & 8785 & 45188 \\
\hline 2015 & 8542 & 44188 \\
\hline 2016 & 8365 & 43463 \\
\hline 2017 & 8218 & 42519 \\
\hline
\end{tabular}

Fuente: Dirección Regional de Salud Amazonas/ Dirección de Estadística y Sistemas de Salud, BD Hechos vitales.

\section{Método, técnica e instrumento}

Se realizó el análisis de documentos correspondientes a los reportes estadísticos y la base de datos de la Dirección de Epidemiología de la Dirección Regional de Salud (DIRESA) - Amazonas de los casos reportados de enfermedades diarreicas agudas (EDA). La información se procesó utilizándose la hoja de cálculo Excel, calculándose la tasa de prevalencia(TP).

$\mathrm{TP}=\frac{\mathrm{N}^{\circ} \text { casos de EDA en un momento dado }}{\text { Total de la población en ese momento }}, 1000$ 


\section{RESULTADOS}

Tabla 1: Tasa de prevalencia de la EDA en niños menores de 5 años de edad en la Región Amazonas. Años 2000-2017.

\begin{tabular}{lccc}
\hline & \multicolumn{3}{c}{ EDA } \\
\cline { 2 - 4 } AÑOS & Acuosa & Disentérica & Total \\
\hline 2000 & 128.5 & 465.0 & 65780.0 \\
2001 & 163.0 & 610.7 & 65906.5 \\
2002 & 145.2 & 513.5 & 70115.9 \\
2003 & 193.5 & 737.9 & 65811.3 \\
2004 & 165.2 & 691.6 & 71165.7 \\
2005 & 202.9 & 754.1 & 70184.1 \\
2006 & 248.0 & 636.1 & 67926.1 \\
2007 & 322.5 & 619.8 & 57613.9 \\
2008 & 313.5 & 337.6 & 55165.0 \\
2009 & 276.0 & 403.2 & 55038.3 \\
2010 & 323.5 & 297.5 & 49817.8 \\
2011 & 296.3 & 323.2 & 49693.4 \\
2012 & 252.1 & 205.8 & 48479.4 \\
2013 & 247.2 & 210.6 & 47758.2 \\
2014 & 270.8 & 170.8 & 46458.4 \\
2015 & 223.1 & 83.4 & 44941.1 \\
2016 & 207.1 & 85.3 & 44293.6 \\
2017 & 202.2 & 58.5 & 43102.7 \\
\hline
\end{tabular}

Fuente: Elaboración propia.

Figura 1: Tendencia de la tasa de prevalencia de la EDA en niños menores de 5 años de edad en la Región Amazonas. Años 2000 - 2017.

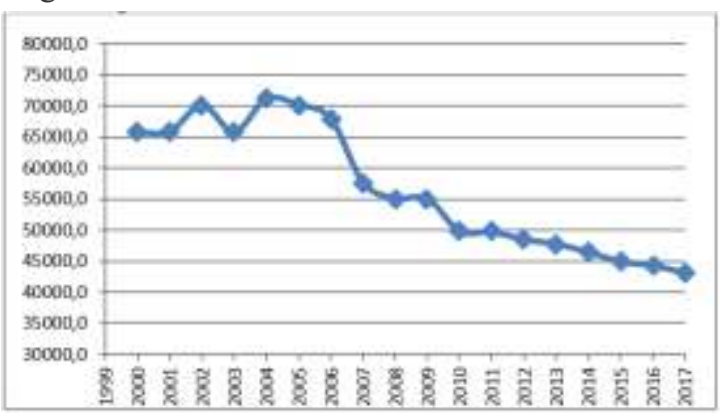

Fuente: Tabla 4.

Tabla 2: Tasa de prevalencia de la EDA en niños menores de 5 años de edad en la provincia de Chachapoyas. Años 2000-2017.

\begin{tabular}{lccc}
\hline & \multicolumn{3}{c}{ EDA } \\
\cline { 2 - 4 } AÑOS & Acuosa & Disentérica & Total \\
\hline 2000 & 10.9 & 9879.0 & 75.7 \\
2001 & 13.6 & 10860.0 & 86.9 \\
2002 & 12.9 & 15022.8 & 67.1 \\
2003 & 16.7 & 12927.6 & 91.9 \\
2004 & 12.6 & 12333.4 & 77.0 \\
2005 & 20.7 & 13318.5 & 118.3
\end{tabular}

\begin{tabular}{lccc}
2006 & 23.5 & 10449.3 & 165.0 \\
2007 & 24.6 & 10978.9 & 145.0 \\
2008 & 23.8 & 3443.9 & 390.3 \\
2009 & 23.3 & 5579.4 & 240.9 \\
2010 & 28.0 & 3217.0 & 445.1 \\
2011 & 22.9 & 2794.1 & 412.3 \\
2012 & 20.9 & 1628.3 & 621.5 \\
2013 & 19.9 & 5227.6 & 195.1 \\
2014 & 33.4 & 1618.1 & 965.3 \\
2015 & 19.5 & 616.6 & 1414.3 \\
2016 & 21.0 & 760.8 & 1222.3 \\
2017 & 16.8 & 952.8 & 766.2 \\
\hline
\end{tabular}

Fuente: Elaboración propia.

Figura 2: Tendencia de la tasa de prevalencia de la EDA en niños menores de 5 años de edad en la Provincia de Chachapoyas. Años 2000-2017.

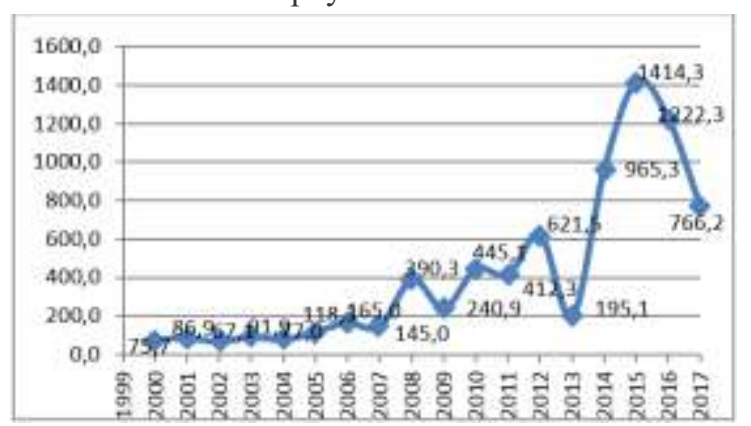

Fuente: Tabla 5.

\section{DISCUSIÓN}

Uno de los pilares que sostiene la vigilancia de en la salud pública lo constituye la detección precoz del comportamiento anormal de los evento de salud, lo cual garantiza que las autoridades sanitarias ofrezcan una intervención oportuna para reducir las consecuencias que pueden ser mortales u causar otros daños. (Miranda \& Ramos, 2010)

El rol de la madre ante el manejo inicial de la diarrea de los niños, es fundamental, es así que en los diferentes estudios realizados en el Perú, reportan prácticas inadecuadas de las madres frente a la diarrea que presentan sus hijos, entre ellos está que restringen la lactancia materna y la alimentación, poca utilización de soluciones de rehidratación oral, y también la automedicación de antibióticos, entre otros. (Guzman, 2016).

Según El Boletín epidemiológico de EsSalud-GCPS - OPIS reportó que en la semana epidemiológica del 29 de enero al 4 de febrero se presentó una tasa ascendente de los episodios de enfermedades diarreicas agudas, en las regiones de Moquegua, Pasco y Amazonas con 105.321 episodios, pudiéndose suponer que la prevalencia de ascenso de debe a múltiples factores clínicos u ambientales. 
La mayoría de los estudios de tendencia en los problemas de salud, se emplea métodos para evaluar las desviaciones del comportamiento actual versus el comportamiento habitual del mismo, está basado en el análisis de series de tiempo que contempla un conjunto de mediciones sobre el estado de una variable o evento de salud considerado, el cual es cronológicamente ordenado. Es así que en el sector de salud, desde hace muchos años, diversos países han orientado sus decisiones en eventos de salud, tal como lo constituye la enfermedad diarreica aguda haciendo uso de modelos de pronósticos, lo cual constituye una herramienta epidemiológica cuyos resultados deben considerarse al momento de plantear políticas de intervención preventiva, lo cual permitirá resultados más eficientes y eficaces. (Miranda \& Ramos, 2010)

Los resultados de la presente investigación muestran que en la Región Amazonas la tasa de prevalencia de la EDA en niños menores de cinco años de edad tuvo tendencia decreciente desde el año 2004 hasta el año 2017, pasando de 71165.7 episodios por cada 1000 infantes en el 2014 a 43102.7 episodios por cada 1000 infantes en el año 2017. La EDA acuosa tuvo tendencia decreciente a partir del año 2010, salvo en el caso del año 2014 que se tuvo un incremento con un valor de 270.8 episodios por cada 1000 infantes, llegando a tener 202.2 episodios en el año 2017. La EDA disentérica tuvo tendencia decreciente para el año 2017 en comparación al año anterior, registrando una tasa de 58.5 episodios por cada 1000 infantes comparado con 85.3 episodios ocurridos en al año 2016. En la Provincia de Chachapoyas, durante los años 2000 al 2017, la EDA tuvo tendencia decreciente a partir del año 2015 hasta el 2017, registrando en estos años una tasa de 1414.3, 1222.2 y 766.2 episodios por cada 1000 infantes respectivamente. La EDA acuosa tuvo tendencia decreciente en el año 2017 en comparación con el año anterior, disminuyendo de 21.0 a 16.8 episodios por cada 1000 infantes, respectivamente. La EDA disentérica tuvo tendencia decreciente hasta el año 2015 que registró 616.6 episodios por cada 1000 infantes, luego del cual se incrementó el siguiente año a 760.8 y siguió incrementándose a 952.8 episodios por cada 1000 infantes en al año 2017.

Estos resultados concuerdan con el estudio de pronóstico de la tendencia nacional y regional de las EDAs en el año 2010, la tendencia de la EDAs en regiones con incremento fueron Ucayali con tasas que oscilaban entre 5059 episodios por cada mil menores de cinco años; en Moquegua las tasas oscilaban 54 y 56; en Arequipa la tasa era de 57 episodios por cada mil menores de cinco años de edad, en Loreto es similar a la de Ucayali con leve descenso al final del año; en la Región La Libertad se estimaron tasas de 30 episodios por cada mil menores de cinco años de edad en los meses de enero y febrero. Las regiones con tendencia al decremento fueron la región de Apurímac con una tasa estimada de 36 episodios por cada mil menores de cinco años en el mes de diciembre, en el Cuzco la tasa estimada fue inicialmente descendente en los meses de junio y julio con 17 episodios por cada mil menores de cinco años de edad, incrementándose nuevamente al final del año; en la Región Madre de Dios se tuvo un incremento progresivo de los casos hasta el mes de agosto con tasas oscilantes entre 57 y 66 episodios, para luego reducir hasta el final de año. Además en este estudio se demuestra que el modelo ARIMA con el enfoque Box-Jenkis mostró que en el año 2010 las EDAs tuvieron una tendencia ascendente en comparación con los años anteriores 2008 y 2009, sugiriéndose que las seis regiones con tendencia al incremento deberían priorizarse las intervenciones en salud. (Miranda \& Ramos, 2010)

Pese a los esfuerzos del sector salud y de la vigilancia epidemiológica que se realice, es importante que se continúe con la vigilancia sanitaria y epidemiológica, y se promueva la capacidad institucional para establecer de manera oportuna no sólo el diagnóstico, sino también el tratamiento adecuado para las enfermedades diarreicas agudas.

\section{CONCLUSIONES}

- En la Región Amazonas la tasa de prevalencia de la EDA en niños menores de cinco años de edad tuvo tendencia decreciente desde el año 2004 hasta el año 2017.

- En la Región Amazonas durante los años 2000 al 2017, la tasa de prevalencia de la EDA acuosa tuvo tendencia decreciente a partir del año 2010, salvo en el caso del año 2014 que se tuvo un incremento.

- En la Región Amazonas durante los años 2000 al 2017, la tasa de prevalencia de la EDA disentérica tuvo tendencia decreciente para el año 2017 en comparación al año anterior.

- En la Provincia de Chachapoyas, durante los años 2000 al 2017, la EDA tuvo tendencia decreciente a partir del año 2015 hasta el 2017 en forma dramática. La EDA acuosa fue decreciente en el año 2017, y la EDA disentérica también fue decreciente hasta el 2015, luego del cual aumentó.

\section{REFERENCIAS BIBLIOGRÁFICAS}

Consejo Nacional de Salud. (8 de julio de 2013). Lineamientos y medidas de reforma del sector salud. Recuperado el 28 de febrero d e $\quad 2017, \quad d e$ http://www.minsa.gob.pe/portada/Especial es/2013/reforma/documentos/documentor 
eforma11122013.pdf

Dirección Regional de Epidemiología. (2013). Análisis de Situación de Salud del Perú. Ministerio de Salud. Lima - Perú: ISBN: 978-9972-820-98-4.

ENDES, I. . (2010). Informe de la Encuesta Demográfica y de Salud familiar. ENDES. Lima.

Gobierno Regional Amazonas. (2010). Plan estratégico institucional 2011 - 2016. Chachapoyas.

Guzman Vera, L. (2016) Prevalencia de enfermedad diarreica aguda en pacientes pediátricos según el plan de atención en la Unidad de rehidratación oral del Hospital Hipólito Unanue. Enero-Marzo 2015. Universidad Ricardo Palma. Lima-Perú.

Hernández Sampieri, R., Fernández, C., \& Baptista, P. (2010). Metodología de la Investigación. México: Mc Graw-Hill.

Hernández, T., Dávila, F., \& Gafanhao, S. (2007). Situación de salud, una herramienta para la gerencia en postgrados. Maracay.

INEI. (2007). Censos nacionales XI de población y VI de vivienda. Instituto Nacional de Estadística e Informática, Lima.

INEI. (2009). Perú: Estimaciones y proyecciones de la población total por años y edades simples 1950 - 2050. Instituto Nacional de Estadística e Informática. Lima: Setiembre.

INEI. (2011). Enfermedades prevalentes de la infancia y su tendencia en los últimos diez años en el Perú. Lima: CIDE.

MINSA. (2016). Boletín epidemiológico del Perú. Semana epidemiológica $\mathrm{N}^{\circ} 20,25$.

Miranda, J. y Ramos Willy (2010). Pronóstico de la tendencia nacional y regional de las enfermedades diarreicas agudas en menores de cinco años de edad en el Perú, mediante un modelo ARIMA con el enfoque Box-Jenkis. Revista Peruana de Epidemiología, vol. 14, num. 1, abril, 2010, pp. 24-31. Sociedad Peruana de Epidemiología. Lima-Perú.

OMS. (2013). Enfermedades diarréicas. Centro de prensa.

OPS. (2009). Manual Clínico para el aprendizaje de AIEPI en Enfermería. Washington D.C.: Biblioteca sede OPS. 